\title{
Dietary patterns and breakfast consumption in relation to insulin resistance in children. The Healthy Growth Study
}

\author{
Kalliopi Karatzi ${ }^{1}$, George Moschonis ${ }^{1}$, Afroditi-Alexandra Barouti ${ }^{1}$, Christos Lionis ${ }^{2}$, \\ George P Chrousos ${ }^{3}$ and Yannis Manios ${ }^{1, *}$ on behalf of the Healthy Growth Study \\ Group \\ 'Department of Nutrition and Dietetics, Harokopio University of Athens, 70 El. Venizelou Avenue, 17671 \\ Kallithea, Athens, Greece: ${ }^{2}$ Clinic of Social and Family Medicine, School of Medicine, University of Crete, \\ Heraklion, Crete, Greece: ${ }^{3}$ King Abdulaziz University, Jeddah, Saudi Arabia
}

Submitted 28 March 2013: Final revision received 23 September 2013: Accepted 8 November 2013: First published online 15 January 2014

\begin{abstract}
Objective: Insulin resistance is a significant cross-point for the manifestation of several chronic diseases in children and adults. The aim of the present study was to investigate the possible relationship of certain dietary patterns and breakfast consumption habits with insulin resistance in children.

Subjects: A representative sample of 1912 schoolchildren (aged 9-13 years) participated in a cross-sectional epidemiological study, the Healthy Growth Study, which was initiated in May 2007 and completed in June 2009.

Setting: It was conducted in seventy-seven primary schools in four large regions in Greece.

Design: Dietary intake, breakfast consumption, anthropometric and physical examination data, biochemical indices and socio-economic information collected from parents were assessed in all children. Principal components analysis was used to identify dietary patterns.

Results: A dietary pattern of increased consumption of margarine, sweets (candies, lollipops, jellies, traditional fruit in heavy syrup) and savoury snacks (chips, cheese puffs and not home-made popcorn) was associated with homeostasis model assessment of insulin resistance index (HOMA-IR; $\beta=0 \cdot 08, P<0 \cdot 001$ ) in multivariate models. Children in the third tertile of this dietary pattern had a $2.51(95 \%$ CI $1 \cdot 30,4 \cdot 90)$ times higher risk of insulin resistance (HOMA-IR $>3 \cdot 16$ ) than those in the first tertile. Breakfast consumption had an inverse correlation with insulin resistance, but the correlation lost its significance after adjustments for waist circumference, birth weight, parental BMI and socio-economic status.

Conclusions: Increased consumption of margarine, sweets and savoury snacks, which is a common dietary pattern in childhood, was positively associated with insulin resistance, while breakfast consumption had an inverse association with HOMA-IR, in schoolchildren (aged 9-13 years). Identification of dietary behaviours that might affect insulin resistance in children offers valuable advice in cardiometabolic risk prevention strategies.
\end{abstract}

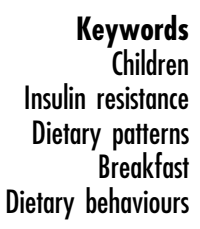

Insulin resistance is an important underlying condition and a critical link between adiposity and increased chronic disease risk, given its association with type 2 diabetes and the metabolic syndrome in both children and adults ${ }^{(1,2)}$. There is a high prevalence of insulin resistance in obese adolescents ${ }^{(1)}$; however, it is also important to investigate the possible role of dietary behaviours in relation to insulin resistance in healthy children and adolescents as well ${ }^{(3)}$. Identification and modification of potentially unfavourable dietary behaviours might be an easy-to-follow method for improving dietrelated health problems.
One dietary behaviour that is usually investigated among children and adolescents is breakfast consumption. Skipping breakfast has been reported to be an index of erratic dietary patterns during the day which consequently can adversely affect cardiometabolic risk factors ${ }^{(4)}$. Irregular breakfast intake has been associated with increased waist circumference and obesity in children ${ }^{(5)}$. Additionally, skipping breakfast in both childhood and adulthood is associated with larger waist circumference and higher fasting insulin levels ${ }^{(6)}$, while in female adolescent girls skipping breakfast is also related to an increased prevalence of insulin resistance ${ }^{(7)}$. 
Furthermore, dietary patterns have been shown to be useful for assessing relationships between diet and chronic diseases ${ }^{(8)}$. Lately they are widely used due to the understanding that nutrients are rarely eaten in isolation and that nutrient-only investigations underestimate the possible interactions between nutrients or between single foods and other dietary components ${ }^{(9)}$. Food is usually consumed as meals consisting of a variety of foods and, therefore, simple consideration of nutrient intake ignores the interaction of various food combinations. Dietary patterns represent the types and amounts of foods consumed and focus on the entire diet rather than just a single food or nutrient ${ }^{(9)}$. Recent data have shown that dietary patterns characterized by high intakes of low-fat dairy products, fruit, whole grains, poultry, fish and vegetables and low intake of alcohol have an inverse relationship with insulin resistance defined by use of the homeostasis model assessment of insulin resistance index (HOMA-IR) in adults ${ }^{(10,11)}$. Likewise, a 'healthy' dietary pattern, rich in whole grains, vegetables and fruits and low in processed meats, high-fat dairy products and sugar, was inversely correlated with insulin resistance in children and adolescents ${ }^{(3,12)}$.

Identification of dietary patterns and behaviours, such as breakfast consumption and snacking choices, associated with insulin resistance could be useful in future interventions for improving lifestyle ${ }^{(13)}$. Although insulin resistance has been linked to breakfast skipping and both have been implicated in chronic diseases ${ }^{(6,7)}$, there is no study investigating dietary patterns and breakfast consumption habits in relation to insulin resistance, particularly among children and adolescents.

Therefore, the aim of the present study was to investigate the possible relationship of certain dietary patterns comprising the consumption of specific food groups/ items and breakfast consumption habits with insulin resistance in children.

\section{Experimental methods}

\section{Sampling}

The Healthy Growth Study was a large-scale, cross-sectional epidemiological study conducted from May 2007 to June 2009. The study was approved by the Greek Ministry of National Education and the Ethics Committee of Harokopio University of Athens and it was in accordance with the ethical standards of the Declaration of Helsinki. The study participants were schoolchildren aged 9-13 years attending primary schools within the counties of Attica, Aitoloakarnania, Thessaloniki and Iraklio. The sampling of schools was random, multistage and stratified by parents' educational level and total population of students attending schools within municipalities of these counties, as described in more detail elsewhere ${ }^{(14)}$. The sampling procedures took into consideration the urban-rural distribution of the population in the four regions, sex and the parental educational level, which is one of the most important indices of socio-economic status (SES). The study population was representative of the 9-13-year-old schoolchildren living in the four counties under study. None the less, these counties are scattered throughout the Greek territory, covering the northern (i.e. Thessaloniki), central (i.e. Attica), western (i.e. Aitoloakarnania) and southern (i.e. Iraklio-Crete) parts of Greece. This, combined with the random, multistage and stratified sampling procedures followed to recruit our sample, is indicative of the representativeness of our population. Written informed consent was obtained from all subjects. Parents who agreed to the participation of their children in the study had to sign the consent form and provide their contact details. Signed parental consent forms were collected for 2656 out of 4145 children (response rate of $64 \cdot 1 \%$ ).

\section{Dietary intake}

Dietary intake data were obtained using the $24 \mathrm{~h}$ recall technique, for two consecutive weekdays and one weekend day. Children were asked to report, as detailed as possible, the type and amount of foods and beverages consumed during the previous day. Interviews with children were conducted by experienced dietitians who had undergone central and rigorous training in order to conduct the $24 \mathrm{~h}$ recalls in a standardized way so as to avoid inter-observer variations. At the end of each interview, trained dietitians reviewed the collected data with the respondent in order to clarify entries, servings and possible forgotten foods. Food intake data were analysed using the Nutritionist $\mathrm{V}$ diet analysis software version $2 \cdot 1$ (First Databank, San Bruno, CA, USA), which was extensively amended to include traditional Greek recipes ${ }^{(15)}$. Furthermore, the database was updated with nutritional information of processed foods provided by independent research institutes, food companies and fast-food chains.

The food-grouping scheme was designed for all foods or entries (core and recipe) appearing in Nutritionist V. In total, forty-seven food groups were initially established, based on similar source characteristics and nutrient contents. Composite food items, such as recipes, were analysed and assigned to food groups according to primary ingredients. A similar methodology for the extraction of food groups was previously reported in studies with a smaller sample size, but with only one $24 \mathrm{~h}$ recall available ${ }^{(16)}$. Examples of foods included in the food groups were documented previously $^{(17)}$.

\section{Breakfast}

Using data from the $24 \mathrm{~h}$ recalls, the portions of each food group were distributed to meals during the day (i.e. breakfast, morning snack, lunch, afternoon snack, dinner, before-bed snack). We considered as breakfast the first things the participant ate and/or drank within $2 \mathrm{~h}$ after getting up in the morning. This could be at home, on 


\begin{tabular}{lcccc}
\hline & \multicolumn{3}{c}{ Score } \\
\cline { 2 - 5 } Component & 0 & 1 & 2 & 3 \\
\hline Parental educational level (years of education) & $<9$ & $9-12$ & $12-16$ & $>16$ \\
Home square metres ( $m^{2}$ ) per family member & $<20$ & $20-25$ & $25-30$ & 2 \\
Number of cars owned by the family & 0 & 1 & $\geq 30$ \\
Home ownership & No & Yes & \\
\hline
\end{tabular}

the way to school or just before entering school. During the weekend, breakfast was considered as anything the participant drank and/or ate before 11.00 hours. Participants were characterized as 'breakfast eaters' based on whether they had breakfast on all three typical days as recorded from the $24 \mathrm{~h}$ recalls. On the other hand, participants who did not have any portion of food and/or drink at breakfast on at least one out of three days were characterized as 'breakfast skippers'.

\section{Antbropometry and physical examination}

Participants underwent a physical examination by two trained members of the research team. The protocol and equipment used were the same in all schools. Weight was measured to the nearest $10 \mathrm{~g}$ using a Seca digital scale (Seca Alpha, Model 770, Hamburg, Germany). Students were weighed without shoes in the minimum clothing possible. Height was measured to the nearest $0 \cdot 1 \mathrm{~cm}$ using a commercial stadiometer (Leicester Height Measure, Invicta Plastics, Oadby, UK) with the participant standing barefoot, keeping shoulders in a relaxed position, arms hanging freely and head in the Frankfort horizontal plane. The cutoff points of the International Obesity Task Force ${ }^{(18,19)}$ were used to categorize participants as 'underweight', 'normal weight', 'overweight' or 'obese'. Waist circumference was measured to the nearest $0 \cdot 1 \mathrm{~cm}$ with the use of a non-elastic tape (Hoechstmass, Germany) with the student standing, at the end of a gentle expiration. Furthermore, one well-trained and experienced female paediatrician in each prefecture determined pubertal maturation (Tanner stage) after thorough visual inspection of breast development in girls and genital development in boys ${ }^{(20)}$.

\section{Biochemical indices}

Blood samples were obtained between 08.30 and 10.30 hours after a $12 \mathrm{~h}$ overnight fast. Part of the blood was collected in test-tubes with no added anticoagulant, where it was allowed to clot for approximately $2 \mathrm{~h}$, as this was designated for serum separation. Clotted blood was centrifuged at $3000 \mathrm{rpm}$ for $15 \mathrm{~min}$ and the collected serum was divided into aliquots and stored at $-80^{\circ} \mathrm{C}$.

Fasting glucose $\left(G_{F}\right)$ was determined using a commercially available enzymatic colorimetric assay (Roche Diagnostics SA, Vasilia, Switzerland). Fasting insulin $\left(\mathrm{I}_{\mathrm{F}}\right)$ was determined using a chemiluminescence immunoassay (Kyowa Medex Ltd, Minami-Ishiki, Japan) for Siemens
Diagnostics USA. Hyperinsulinaemia was considered when children had blood insulin values of $>20 \mu \mathrm{U} / \mathrm{ml}^{(21)}$. Insulin resistance was assessed through homeostasis model assessment ${ }^{(22)}$, calculated using $\mathrm{G}_{\mathrm{F}}$ and $\mathrm{I}_{\mathrm{F}}$, as follows: HOMA-IR $=\left[\mathrm{I}_{\mathrm{F}}(\mu \mathrm{U} / \mathrm{ml}) \times \mathrm{G}_{\mathrm{F}}(\mathrm{mmol} / \mathrm{l})\right] / 22 \cdot 5$, which is a valid tool for assessing insulin resistance in children and adolescents $^{(23)}$. As no widely acceptable definition has been proposed for insulin resistance, we used a threshold for HOMA-IR (HOMA-IR $>3 \cdot 16)^{(23)}$ which is validated in children and adolescents.

\section{Socio-economic, perinatal and otber information collected from the parents}

Data on the socio-economic background of the families were collected from the parents (most preferably from the mother) during scheduled face-to-face interviews at school. For those parents not able to attend the meetings (approximately $5 \%$ of the total sample), data were collected via telephone interviews. The socio-economic data included parental educational level (years of education); home square metres $\left(\mathrm{m}^{2}\right)$; number of family members living at home; number of cars owned by the family; and home ownership. All aforementioned data were grouped, scored and combined for the development of an index of SES (SES index) as indicated in Table 1. The total score of the SES index was obtained by summing the scores for each index component. The values of the total SES index score ranged between 0 and 13, with higher values indicating higher SES of the family. The self-reported weight and height of the mother and the father were also recorded. Paediatric records were used to obtain data about children's birth weight.

\section{Physical activity assessment via step count}

To assess step count as an objective estimate of physical activity, study participants were provided with and instructed to wear a waist-mounted pedometer (Yamax SW-200 Digiwalker, Tokyo, Japan) for one week, i.e. from Monday to Sunday. The pedometer was positioned according to the manufacturer's instructions on the right of the waistband, vertically aligned with the patella. Children were instructed to wear the pedometer from the time they woke up in the morning until the time they went to bed at night (except when taking a shower, bathing or swimming) and to record their total number of daily steps displayed by the pedometer in a diary template before bedtime. 


\section{Statistical analysis}

Dietary component derivation

Principal components analysis (PCA) was used to identify dietary patterns. The Kaiser-Meyer-Olkin (KMO) criterion was applied and it was equal to 0.52 . The orthogonal rotation (varimax option) was used to derive optimal non-correlated components (dietary patterns). To decide the number of components to retain, the Kaiser criterion (eigenvalues $>1$ ) was used. Factor loadings represent the correlations of each food or food group with the dietary pattern score. Higher absolute values of factor loadings indicate that the food or food group predictor contributes most to the construction of this particular component. The dietary components (patterns) were named according to the factor loadings of those foods or food groups correlated most with the component (factor loadings $>|0.4|)$. When a behaviour had a factor loading value $>|0.4|$ in more than one component, then this food or food group was used to name the component for which it had the higher factor loading value.

\section{Descriptive and other statistical analyses}

Categorical variables were presented as frequencies. Associations between categorical variables were examined by using the $\chi^{2}$ test and the two-sample $z$ test for proportions whenever appropriate. Multiple linear regression analysis was used to evaluate the associations between insulin resistance (HOMA-IR $>3 \cdot 16$ ), breakfast consumption habits and dietary patterns derived from PCA, which were treated as continuous variables. More specifically, three different models were applied: model 1 was unadjusted, model 2 was adjusted for sex and Tanner stage, and model 3 was adjusted for sex, Tanner stage, waist circumference, mean parental BMI, SES index score and birth weight. The results from the linear regression models are presented as standardized beta coefficients and the level of significance was defined at $P<0.05$. Breakfast consumption was used as a categorical variable with 0 representing breakfast skippers and 1 representing breakfast eaters. Moreover, participants' dietary pattern scores were categorized into tertiles so that, for each dietary component, tertile 3 consisted of persons whose dietary intake most adhered to that particular pattern. Based on the statistically significant associations provided by the linear regression models, logistic regression analyses were performed to evaluate the association between the tertiles of each dietary component and the probability of insulin resistance. The results of logistic regression models were presented as odds ratios and 95\% confidence intervals. Data were analysed using the statistical software package SPSS version $13 \cdot 0$.

\section{Results}

Full socio-economic, demographic, parental BMI, perinatal, clinical, biochemical and dietary data were collected for 1912 children $(50 \cdot 1 \%$ females and $49 \cdot 9 \%$ males). Regarding the descriptive characteristics of the study sample, Table 2 illustrates that the total prevalence of overweight and obesity was $30 \cdot 8 \%$ and $11 \cdot 7 \%$, respectively, with a significantly higher prevalence of obesity in boys than in girls $(14 \cdot 2 \% v \cdot 9 \cdot 2 \%, P<0 \cdot 05)$. Furthermore, Table 2 shows that the prevalence of insulin resistance (i.e. HOMA-IR $>3 \cdot 16$ ) was significantly higher in girls than in boys $(33.6 \% v .23 .4 \%, P<0.05)$.

Table 2 Anthropometric, clinical and biochemical characteristics of the study population and prevalence of central obesity, insulin resistance and hyperinsulinaemia: representative sample of 1912 Greek schoolchildren aged 9-13 years, Healthy Growth Study, May 2007-June 2009

\begin{tabular}{|c|c|c|c|c|c|c|}
\hline & \multicolumn{2}{|c|}{ Boys ( $n$ 930) } & \multicolumn{2}{|c|}{ Girls (n 982) } & \multicolumn{2}{|c|}{ Total (n 1912) } \\
\hline & Mean & SD & Mean & SD & Mean & SD \\
\hline Age (years) & $11 \cdot 1$ & $0 \cdot 7$ & $11 \cdot 2$ & 0.7 & $11 \cdot 1$ & $0 \cdot 7$ \\
\hline Weight (kg) & $45 \cdot 8$ & $11 \cdot 1$ & $45 \cdot 0$ & $10 \cdot 7$ & $45 \cdot 4$ & $10 \cdot 9$ \\
\hline Height (cm) & $148 \cdot 3^{*}$ & $7 \cdot 4$ & $149 \cdot 1$ & $8 \cdot 0$ & $148 \cdot 7$ & $7 \cdot 7$ \\
\hline $\mathrm{WC}(\mathrm{cm})$ & $70 \cdot 2^{*}$ & $10 \cdot 2$ & $67 \cdot 4$ & 8.9 & $68 \cdot 8$ & $9 \cdot 6$ \\
\hline Birth weight (g) & $3293 \cdot 8^{*}$ & $519 \cdot 9$ & $3197 \cdot 4$ & $511 \cdot 1$ & $3244 \cdot 3$ & $517 \cdot 5$ \\
\hline \multicolumn{7}{|l|}{ Weight status (\%) } \\
\hline Underweight & \multicolumn{2}{|c|}{$1 \cdot 8 t$} & \multicolumn{2}{|c|}{$3 \cdot 4$} & \multicolumn{2}{|c|}{$2 \cdot 6$} \\
\hline Normal & \multicolumn{2}{|c|}{$51 \cdot 6+$} & \multicolumn{2}{|c|}{$58 \cdot 1$} & \multicolumn{2}{|c|}{$54 \cdot 9$} \\
\hline Overweight & \multicolumn{2}{|c|}{$32 \cdot 4$} & \multicolumn{2}{|c|}{$29 \cdot 3$} & \multicolumn{2}{|c|}{$30 \cdot 8$} \\
\hline Obese & \multicolumn{2}{|c|}{$14 \cdot 2+$} & \multicolumn{2}{|c|}{$9 \cdot 2$} & \multicolumn{2}{|c|}{$11 \cdot 7$} \\
\hline Normal central body weight distribution (\%) & \multicolumn{2}{|c|}{$83 \cdot 6$} & \multicolumn{2}{|c|}{$86 \cdot 3$} & \multicolumn{2}{|c|}{$85 \cdot 0$} \\
\hline Central obesity (\%) & \multicolumn{2}{|c|}{$16 \cdot 4$} & \multicolumn{2}{|c|}{$13 \cdot 7$} & \multicolumn{2}{|c|}{$15 \cdot 0$} \\
\hline Fasting glucose (mg/dl) & $94 \cdot 0^{*}$ & $10 \cdot 4$ & $91 \cdot 5$ & $9 \cdot 2$ & $92 \cdot 7$ & $9 \cdot 9$ \\
\hline Fasting insulin ( $\mu \mathrm{IU} / \mathrm{ml})$ & $11 \cdot 0^{*}$ & $8 \cdot 7$ & $12 \cdot 9$ & $7 \cdot 3$ & $12 \cdot 0$ & $8 \cdot 0$ \\
\hline HOMA-IR & $2 \cdot 54^{*}$ & $2 \cdot 14$ & $2 \cdot 89$ & $1 \cdot 66$ & $2 \cdot 72$ & $1 \cdot 92$ \\
\hline Insulin resistance (HOMA-IR > 3.16) (\%) & \multicolumn{2}{|c|}{$23 \cdot 4+$} & \multicolumn{2}{|c|}{$33 \cdot 6$} & \multicolumn{2}{|c|}{$28 \cdot 7$} \\
\hline Hyperinsulinaemia (\%) & \multicolumn{2}{|c|}{$6 \cdot 5 t$} & \multicolumn{2}{|c|}{$14 \cdot 0$} & \multicolumn{2}{|c|}{$10 \cdot 2$} \\
\hline
\end{tabular}

HOMA-IR, homeostasis model assessment of insulin resistance index; WC, waist circumference.

${ }^{\star} P<0.05$ for sex differences based on Student's $t$ test.

$+P<0.05$ for sex differences based on the $\chi^{2}$ test and the two-sample $z$ test for proportions for multiple comparisons, where appropriate. 
Table 3 Factor loadings for the five dietary patterns derived from principal components analysis regarding certain food groups in the study population: representative sample of 1912 Greek schoolchildren aged 9-13 years, Healthy Growth Study, May 2007-June 2009

\begin{tabular}{|c|c|c|c|c|c|}
\hline \multirow[b]{2}{*}{ Predictor (food group) } & \multicolumn{5}{|c|}{ Dietary pattern } \\
\hline & 1 & 2 & 3 & 4 & 5 \\
\hline Fried potato & 0.850 & -0.014 & -0.006 & -0.001 & -0.050 \\
\hline Red meat ${ }^{*}$ & 0.806 & 0.026 & 0.000 & 0.014 & -0.081 \\
\hline Sugared beveragest & 0.556 & 0.040 & -0.009 & -0.010 & 0.148 \\
\hline Processed meatsł & 0.050 & 0.840 & -0.010 & -0.092 & -0.135 \\
\hline Cheese§ & 0.011 & 0.835 & -0.027 & $0 \cdot 167$ & 0.054 \\
\hline Margarine & -0.013 & 0.029 & 0.819 & -0.009 & -0.040 \\
\hline Sweets and savoury snacksll & -0.001 & -0.062 & 0.815 & 0.032 & 0.038 \\
\hline Legumes & -0.014 & 0.133 & 0.023 & 0.748 & $0 \cdot 102$ \\
\hline Fruit & 0.013 & -0.061 & 0.001 & 0.731 & -0.064 \\
\hline Egg & 0.016 & $0 \cdot 104$ & 0.011 & -0.139 & 0.830 \\
\hline Fish & -0.020 & 0.147 & 0.010 & -0.136 & -0.510 \\
\hline Explained variance (\%) & $15 \cdot 3$ & $13 \cdot 3$ & $12 \cdot 2$ & $10 \cdot 6$ & $9 \cdot 3$ \\
\hline
\end{tabular}

Variable with the highest factor loading $(>10.41)$ within the component is shown in bold.

*Beef, pork, lamb, goat meat and meat balls.

tCola-type soda, orange and sprite soda.

$\ddagger$ Salami, pariza, turkey ham, pork ham, sausages, bacon and also in croissants and in savoury pies.

\$Hard and semi-hard cheese (gouda/edam, etc.), soft cheese ('anthotyro', a type of cheese similar to ricotta) and also in pies (e.g. cheese pie).

IISweets high in sugar and low in fat (candies, lollipops, jellies, traditional fruit in heavy syrup) and salty snacks such as chips, cheese puffs and popcorn (not home-made)

-Boiled or poached eggs; consumption of boiled or steamed fish.

Table 3 summarizes the loadings of the factors retained from the PCA. The value of the KMO criterion indicates that the dietary variables entered in the analysis were strongly inter-correlated and that PCA could be correctly used for assessing 'healthy' or 'unhealthy' dietary patterns. The PCA indicated five dietary components explaining $60.7 \%$ of the total variance with regard to the examined variables. These components were characterized as follows: fried potato, red meat and sugared beverages (component 1); processed meats and cheese (component 2); margarine, sweets and savoury snacks (component 3); legumes and fruits (component 4); and higher egg and lower fish consumption (component 5).

Table 4 presents the associations derived from the three different models of the multiple linear regression analysis between HOMA-IR (dependent variable), the dietary components and regular breakfast consumption. Dietary component 3 was positively associated with HOMA-IR $(\beta=0 \cdot 15, P<0 \cdot 001 ;$ model 1$)$ and even after adjusting for several potential confounding factors in models 2 and 3 , this association remained statistically significant $(\beta=0 \cdot 17$, $P<0.001$ and $\beta=0 \cdot 08, P<0 \cdot 001$, respectively). Dietary component 5 was also found to be positively associated with HOMA-IR in model $1(\beta=0 \cdot 09, P=0 \cdot 02)$ but lost its significance after adjusting for several confounders (models 2 and 3). Regular breakfast consumption was inversely associated with HOMA-IR in model 1 and model 2, but lost its significance in model 3 for each dietary component (Table 4).

The logistic regression analysis summarized in Table 5 showed that the third tertile of dietary component 3 was significantly associated with the incidence of insulin resistance. Specifically, children who showed greater adherence to dietary component 3 (highest tertile) were $2 \cdot 51$ (95\% CI $1 \cdot 30,4 \cdot 90)$ times more likely to have insulin resistance (based on HOMA-IR >3·16) compared with children in the first tertile, after controlling for several potential confounding factors.

\section{Discussion}

The prevalence of insulin resistance, obesity and diabetes in children and adolescents is constantly increasing ${ }^{(24)}$, with overweight and obese children being more insulin resistant than normal-weight children. It is evident that these conditions increase the risk of developing the metabolic syndrome, CVD and diabetes in adulthood ${ }^{(25)}$. Therefore it is essential to identify factors related to diet and lifestyle in childhood as an attempt to modify the risk of unfavourable health outcomes later in life ${ }^{(26)}$.

In terms of anthropometric indices, concerning the prevalence of overweight and obesity shown in Table 2, our study results are in accordance with proportional results from previous studies in both European ${ }^{(27)}$ and Greek children ${ }^{(28-30)}$, introducing obesity levels of about $5-14 \%$ and overweight levels of about $20-31 \%$.

The PCA conducted in the present study revealed predominantly five dietary components. Out of these, only component 3 comprising higher consumption of margarine, sweets (i.e. candies, lollipops, jellies, traditional fruit in heavy syrup) and savoury snacks (i.e. chips, cheese puffs and not home-made popcorn) was significantly associated with HOMA-IR after adjustment for several potential confounders. This dietary behaviour is very common in children and adolescents and has been previously described in studies concerning the food preferences of children and adolescents, which showed that nearly $40 \%$ of total energy consumed by $2-18$-year-olds 
Table 4 Associations of different dietary patterns and breakfast consumption with HOMA-IR in the study population: representative sample of 1912 Greek schoolchildren aged 9-13 years, Healthy Growth Study, May 2007-June 2009

\begin{tabular}{|c|c|c|c|c|c|c|}
\hline \multirow[b]{2}{*}{ HOMA-IR (dependent variable) } & \multicolumn{2}{|c|}{ Model 1} & \multicolumn{2}{|c|}{ Model 2} & \multicolumn{2}{|c|}{ Model 3} \\
\hline & $\beta$ & $P$ value & $\beta$ & $P$ value & $\beta$ & $P$ value \\
\hline \multicolumn{7}{|c|}{$\begin{array}{l}\text { Independent variables in each model } \\
\text { Model A }\end{array}$} \\
\hline $\begin{array}{l}\text { Dietary pattern } 1 \\
\text { Breakfast consumption }\end{array}$ & $\begin{array}{l}-0.02 \\
-0.41\end{array}$ & $\begin{aligned} & 0.53 \\
< & 0.001\end{aligned}$ & $\begin{array}{l}-0.01 \\
-0.37\end{array}$ & $\begin{aligned} & 0.70 \\
< & 0.001\end{aligned}$ & $\begin{array}{r}0.01 \\
-0.03\end{array}$ & $\begin{array}{l}0 \cdot 78 \\
0 \cdot 12\end{array}$ \\
\hline \multicolumn{7}{|l|}{ Model B } \\
\hline $\begin{array}{l}\text { Dietary pattern } 2 \\
\text { Breakfast consumption }\end{array}$ & $\begin{array}{r}0.03 \\
-0.41\end{array}$ & $\begin{aligned} & 0.53 \\
< & 0.001\end{aligned}$ & $\begin{array}{r}0.01 \\
-0.37\end{array}$ & $\begin{aligned} & 0.72 \\
< & 0.001\end{aligned}$ & $\begin{array}{l}-0.05 \\
-0.03\end{array}$ & $\begin{array}{l}0 \cdot 81 \\
0 \cdot 12\end{array}$ \\
\hline \multicolumn{7}{|l|}{ Model C } \\
\hline $\begin{array}{l}\text { Dietary pattern } 3 \\
\text { Breakfast consumption }\end{array}$ & $\begin{array}{r}0.15 \\
-0.43\end{array}$ & $\begin{array}{l}<0.001 \\
<0.001\end{array}$ & $\begin{array}{r}0 \cdot 17 \\
-0 \cdot 39\end{array}$ & $\begin{array}{l}<0.001 \\
<0.001\end{array}$ & $\begin{array}{r}0.08 \\
-0.04\end{array}$ & $\begin{array}{c}<0.001 \\
0.82\end{array}$ \\
\hline \multicolumn{7}{|l|}{ Model D } \\
\hline $\begin{array}{l}\text { Dietary pattern } 4 \\
\text { Breakfast consumption }\end{array}$ & $\begin{array}{l}-0.03 \\
-0.41\end{array}$ & $\begin{array}{c}0.43 \\
<\mathbf{0 . 0 0 1}\end{array}$ & $\begin{array}{l}-0.03 \\
-0.37\end{array}$ & $\begin{array}{l}0.46 \\
<\mathbf{0 . 0 0 1}\end{array}$ & $\begin{array}{l}-0.01 \\
-0.03\end{array}$ & $\begin{array}{l}0 \cdot 78 \\
0 \cdot 12\end{array}$ \\
\hline \multicolumn{7}{|l|}{ Model E } \\
\hline $\begin{array}{l}\text { Dietary pattern } 5 \\
\text { Breakfast consumption }\end{array}$ & $\begin{array}{r}0.09 \\
-0.41\end{array}$ & $\begin{array}{c}0.02 \\
<0.001\end{array}$ & $\begin{array}{r}0.07 \\
-0.37\end{array}$ & $\begin{aligned} & 0.08 \\
< & 0.001\end{aligned}$ & $\begin{array}{r}0.02 \\
-0.03\end{array}$ & $\begin{array}{l}0.25 \\
0 \cdot 13\end{array}$ \\
\hline
\end{tabular}

HOMA-IR, homeostasis model assessment of insulin resistance index; $\beta$, standardized beta coefficient; WC, waist circumference; SES, socio-economic status.

Significant results are shown in bold font.

Model $1=$ adjusted for breakfast consumption. Model $2=$ adjusted for breakfast consumption, sex and Tanner stage. Model $3=$ adjusted for breakfast consumption, sex, Tanner stage, WC, parental BMI, SES index, birth weight and physical activity.

Breakfast consumption = eating $v$. skipping. Dietary pattern $1=$ higher consumption of fried potatoes, red meat and sugared beverages. Dietary pattern 2 = higher consumption of processed meat and cheese. Dietary pattern $3=$ higher consumption of margarine and higher consumption of sweets and savoury snacks. Dietary pattern 4 = higher fruit and legumes consumption. Dietary pattern $5=$ higher egg consumption and lower fish consumption.

Table 5 Logistic regression analysis examining the correlation between tertiles of dietary pattern 3 and the likelihood of insulin resistance in the study population: representative sample of 1912 Greek schoolchildren aged 9-13 years, Healthy Growth Study, May 2007-June 2009

\begin{tabular}{|c|c|c|c|c|c|}
\hline \multirow{3}{*}{$\begin{array}{l}\text { Insulin resistance based on } \\
\mathrm{HOMA}-\mathrm{IR}>3 \cdot 16 \text { (dependent variable) }\end{array}$} & \multicolumn{5}{|c|}{ Tertile of dietary pattern 3 (independent variable) } \\
\hline & \multirow[b]{2}{*}{ 1st tertile } & \multicolumn{2}{|c|}{ 2nd tertile } & \multicolumn{2}{|c|}{ 3rd tertile } \\
\hline & & OR & $95 \% \mathrm{Cl}$ & OR & $95 \% \mathrm{Cl}$ \\
\hline Model 1 & $1 \cdot 00$ & $1 \cdot 37$ & $0 \cdot 83,2 \cdot 26$ & 1.66 & $1 \cdot 00,2 \cdot 78$ \\
\hline Model 2 & 1.00 & 1.58 & $0.93,2 \cdot 71$ & 1.94 & $1 \cdot 12,3 \cdot 36$ \\
\hline Model 3 & 1.00 & 1.91 & $1 \cdot 00,3.65$ & $2 \cdot 51$ & $1 \cdot 30,4 \cdot 90$ \\
\hline
\end{tabular}

HOMA-IR, homeostasis model assessment of insulin resistance index; WC, waist circumference; SES, socio-economic status.

Significant results are shown in bold font.

Dietary pattern $3=$ higher consumption of margarine and higher consumption of sweets and savoury snacks.

Model $1=$ adjusted for breakfast consumption. Model $2=$ adjusted for breakfast consumption, sex and Tanner stage. Model $3=$ adjusted for breakfast consumption, sex, Tanner stage, WC, parental BMI, SES index, birth weight and physical activity.

was 'empty calories' coming from desserts, added sugars, whole milk and pizza ${ }^{(31)}$.

Studies concerning dietary fat intake and insulin resistance have shown inconsistent results, mainly due to several study flaws that limit the validity of their findings ${ }^{(32)}$. The main outcome coming from correlations between the quality of fat intake and insulin resistance is either that there is no significant association or that there is an inverse association between saturated fat consumption and insulin sensitivity ${ }^{(33)}$. It seems that diets rich in SFA reduce insulin sensitivity in healthy adults ${ }^{(34)}$, which is in line with our findings showing that a dietary behaviour that included foods rich in saturated fat (chips and cheese puffs) is significantly associated with insulin resistance. However, these previous studies were conducted mainly in healthy or diabetic adults and only one relevant study in children has shown that increased total fat intake, regardless of its quality, is inversely associated with insulin sensitivity in black but not in white children ${ }^{(35)}$.

Additionally, it was shown previously that sugar intake in the form of sugar-sweetened beverages was associated with insulin resistance in adolescents ${ }^{(12,36)}$. Data from children and adolescents showed that total sugar intake is associated with insulin resistance in girls, but not boys ${ }^{(12,36)}$. It is already known that the short absorption time that follows the consumption of sugar may impair blood glucose control, which may result in hyperinsulinaemia and peripheral insulin resistance ${ }^{(36)}$. Our results are in accordance with the above-mentioned findings as the dietary behaviour which was related to insulin resistance included candies, lollipops, jellies and traditional fruit in heavy syrup, all foods with a high glycaemic index. 
Regarding breakfast, it seems that many adolescents in Western countries skip breakfast with a prevalence reaching even $34 \%{ }^{(37)}$. Skipping breakfast has been associated with overweight and obesity, as well as increased fasting insulin and insulin resistance in children and adolescents $^{(4,6,7,38)}$. In our study there was a strong inverse association between breakfast eating and insulin resistance even after adjustment for gender and Tanner stage, which is in accordance with the aforementioned studies. However, this association lost its significance when parental BMI, SES index, waist circumference and birth weight were entered in the multivariate analysis, as additional covariates. It is already evident that parental weight status, children's birth weight and most socio-economic data constituting the SES index, like parental education level, are associated with childhood obesity ${ }^{(14,39)}$. Additionally, childhood obesity assessed through waist circumference and other anthropometric indices is associated with insulin resistance and specifically HOMA-IR ${ }^{(40,41)}$. Therefore, according to our data, breakfast consumption is inversely associated with insulin resistance. However this association is confounded by indices related to childhood obesity.

The current study has both strengths and limitations. The Healthy Growth Study was a large-scale epidemiological study conducted in a representative sample of children from four prefectures within the wider region of Greece. Furthermore, the adjustment for specific confounders resulted in the extraction of more accurate results. However, as the study has a cross-sectional design, it is not suitable for interpreting causal relationships. Additionally, use of parental BMI coming from self-reported height and weight as a confounder could be another limitation. Finally, it should be noted that use of self-reported $24 \mathrm{~h}$ recalls is a subjective measure of dietary intake.

\section{Conclusion}

A dietary pattern comprising increased consumption of margarine, sweets (candies, lollipops, jellies) and savoury snacks (chips, cheese puffs) was positively associated with insulin resistance in children aged 9-13 years, while breakfast consumption was inversely associated with HOMA-IR, but lost its significance after adjustment for indices related to childhood obesity. This finding could be taken into consideration when developing public health prevention programmes in order to reduce cardiometabolic risk factors and future unfavourable health outcomes in adulthood. However, more studies are needed in order to explore the association of dietary patterns with insulin resistance in children.

\section{Acknowledgements}

Sources of funding: This research has been co-financed by the European Union (European Social Fund - ESF) and Greek national funds through the Operational Programme 'Education and Lifelong Learning' of the National Strategic Reference Framework (NSRF) - Research Funding Programme 'Heracleitus II. Investing in knowledge society through the European Social Fund'. Neither the European Union nor the Greek national funding had any role in the design, analysis or writing of this article. Conflicts of interest: There is no conflict of interest for any of the authors. Authors' contributions: K.K., analysis and/or interpretation of data, drafting/revision of the manuscript, approval of the final version of the manuscript. G.M., conception and design of the study, collection, analysis and/or interpretation of data, drafting/revision of the manuscript, approval of the final version of the manuscript. A.-A.B., analysis and/or interpretation of data, drafting/ revision of the manuscript. C.L., interpretation of data, drafting/revision of the manuscript, approval of the final version of the manuscript. G.P.C., interpretation of data, drafting/revision of the manuscript, approval of the final version of the manuscript. Y.M., conception and design of the study, interpretation of data, drafting/revision of the manuscript, approval of the final version of the manuscript.

\section{References}

1. Lee JM (2006) Insulin resistance in children and adolescents. Rev Endocr Metab Disord 7, 141-147.

2. Seidell JC (2000) Obesity, insulin resistance and diabetes a worldwide epidemic. Br J Nutr 83, Suppl. 1, S5-S8.

3. Ambrosini GL, Huang RC, Mori TA et al. (2010) Dietary patterns and markers for the metabolic syndrome in Australian adolescents. Nutr Metab Cardiovasc Dis 20, 274-283.

4. Farshchi HR, Taylor MA \& Macdonald IA (2005) Deleterious effects of omitting breakfast on insulin sensitivity and fasting lipid profiles in healthy lean women. Am J Clin Nutr 81, 388-396.

5. Lehto R, Ray C, Lahti-Koski M et al. (2011) Health behaviors, waist circumference and waist-to-height ratio in children. Eur J Clin Nutr 65, 841-848.

6. Smith KJ, Gall SL, McNaughton SA et al. (2010) Skipping breakfast: longitudinal associations with cardiometabolic risk factors in the Childhood Determinants of Adult Health Study. Am J Clin Nutr 92, 1316-1325.

7. Sese MA, Jimenez-Pavon D, Gilbert CC et al. (2012) Eating behaviour, insulin resistance and cluster of metabolic risk factors in European adolescents. The HELENA Study. Appetite 59, 140-147.

8. Hu FB (2002) Dietary pattern analysis: a new direction in nutritional epidemiology. Curr Opin Lipidol 13, 3-9.

9. Jacques PF \& Tucker KL (2001) Are dietary patterns useful for understanding the role of diet in chronic disease? $\mathrm{Am} \mathrm{J}$ Clin Nutr 73, 1-2.

10. Akter S, Nanri A, Yi S et al. (2012) Dietary patterns and Cpeptide concentrations in a Japanese working population. Nutrition 28, e29-e35.

11. Anderson AL, Harris TB, Tylavsky FA et al. (2012) Dietary patterns, insulin sensitivity and inflammation in older adults. Eur J Clin Nutr 66, 18-24.

12. Kynde I, Johnsen NF, Wedderkopp N et al. (2010) Intake of total dietary sugar and fibre is associated with insulin resistance among Danish 8-10- and 14-16-year-old girls but not boys. European Youth Heart Studies I and II. Public Health Nutr 13, 1669-1674. 
13. Owen K, Pettman T, Haas $M$ et al. (2010) Individual preferences for diet and exercise programmes: changes over a lifestyle intervention and their link with outcomes. Public Health Nutr 13, 245-252.

14. Moschonis G, Tanagra S, Vandorou A et al. (2010) Social, economic and demographic correlates of overweight and obesity in primary-school children: preliminary data from the Healthy Growth Study. Public Health Nutr 13, $1693-1700$

15. Trichopoulou A (2004) Composition Tables of Foods and Greek Dishes. Athens: Department of Hygiene and Epidemiology, School of Medicine, University of Athens.

16. Nicklas TA, Webber LS, Srinivasan SR et al. (1993) Secular trends in dietary intakes and cardiovascular risk factors of 10-y-old children: the Bogalusa Heart Study (1973-1988). Am J Clin Nutr 57, 930-937.

17. Nicklas TA, Farris RP, Johnson CC et al. (1990) Food Sources of Nutrients: A Tool for Dietary Management and Health. The Bogalusa Heart Study 1973-1983. New Orleans, LA: National Center for Cardiovascular Health, Louisiana State University Medical Center.

18. Cole TJ, Bellizzi MC, Flegal KM et al. (2000) Establishing a standard definition for child overweight and obesity worldwide: international survey. BMJ 320, 1240-1243.

19. Cole TJ, Flegal KM, Nicholls D et al. (2007) Body mass index cut offs to define thinness in children and adolescents: international survey. BMJ 335, 194.

20. Tanner JM (1955) Growth at Adolescence. Oxford: Blackwell Scientific.

21. Kratz A, Ferraro M, Sluss PM et al. (2004) Case records of the Massachusetts General Hospital. Weekly clinicopathological exercises. Laboratory reference values. $N$ Engl J Med 351, 1548-1563.

22. Matthews DR, Hosker JP, Rudenski AS et al. (1985) Homeostasis model assessment: insulin resistance and $\beta$-cell function from fasting plasma glucose and insulin concentrations in man. Diabetologia 28, 412-419.

23. Conwell LS, Trost SG, Brown WJ et al. (2004) Indexes of insulin resistance and secretion in obese children and adolescents: a validation study. Diabetes Care 27, 314-319.

24. van Vliet M, Heymans MW, von Rosenstiel IA et al. (2011) Cardiometabolic risk variables in overweight and obese children: a worldwide comparison. Cardiovasc Diabetol 10, 106.

25. Magnussen CG, Koskinen J, Chen W et al. (2010) Pediatric metabolic syndrome predicts adulthood metabolic syndrome, subclinical atherosclerosis, and type 2 diabetes mellitus but is no better than body mass index alone: the Bogalusa Heart Study and the Cardiovascular Risk in Young Finns Study. Circulation 122, 1604-1611.

26. Halfon N, Verhoef PA \& Kuo AA (2012) Childhood antecedents to adult cardiovascular disease. Pediatr Rev 33, 51-60.
27. Brug J, van Stralen MM, Te Velde SJ et al. (2012) Differences in weight status and energy-balance related behaviors among schoolchildren across Europe: the ENERGY-project. PLoS One 7, e34742.

28. Manios Y, Yiannakouris N, Papoutsakis C et al. (2004) Behavioral and physiological indices related to BMI in a cohort of primary schoolchildren in Greece. Am J Hum Biol 16, 639-647.

29. Manios Y, Magkos F, Christakis G et al. (2005) Twenty-year dynamics in adiposity and blood lipids of Greek children: regional differences in Crete persist. Acta Paediatr 94, 859-865.

30. Magkos F, Manios Y, Christakis G et al. (2006) Agedependent changes in body size of Greek boys from 1982 to 2002. Obesity (Silver Spring) 14, 289-294.

31. Reedy J \& Krebs-Smith SM (2010) Dietary sources of energy, solid fats, and added sugars among children and adolescents in the United States. J Am Diet Assoc 110, 1477-1484.

32. Galgani JE, Uauy RD, Aguirre CA et al. (2008) Effect of the dietary fat quality on insulin sensitivity. Br J Nutr 100, 471-479.

33. Levy-Marchal C, Arslanian S, Cutfield W et al. (2010) Insulin resistance in children: consensus, perspective, and future directions. J Clin Endocrinol Metab 95, 5189-5198.

34. Vessby B, Uusitupa M, Hermansen K et al. (2001) Substituting dietary saturated for monounsaturated fat impairs insulin sensitivity in healthy men and women: the KANWU Study. Diabetologia 44, 312-319.

35. Weigensberg MJ, Ball GD, Shaibi GQ et al. (2005) Dietary fat intake and insulin resistance in black and white children. Obes Res 13, 1630-1637.

36. Harrington S (2008) The role of sugar-sweetened beverage consumption in adolescent obesity: a review of the literature. J Sch Nurs 24, 3-12.

37. Rampersaud GC, Pereira MA, Girard BL et al. (2005) Breakfast habits, nutritional status, body weight, and academic performance in children and adolescents. $J \mathrm{Am}$ Diet Assoc 105, 743-760.

38. Moschonis G, Kalliora A, Kostarelli V et al. (2013) Identification of lifestyle patterns associated with obesity and fat mass in children: the Healthy Growth Study. Public Health Nutr (Epublication ahead of print version).

39. Birbilis M, Moschonis G, Mougios V et al. (2013) Obesity in adolescence is associated with perinatal risk factors, parental BMI and sociodemographic characteristics. Eur J Clin Nutr 67, 115-121.

40. Manios Y, Kourlaba G, Kafatos A et al. (2008) Associations of several anthropometric indices with insulin resistance in children: the Children Study. Acta Paediatr 97, 494-499.

41. Manios Y, Moschonis G, Kourlaba G et al. (2008) Prevalence and independent predictors of insulin resistance in children from Crete, Greece: the Children Study. Diabet Med 25, 65-72. 